\title{
Current review of surgical management options for total knee arthroplasty in the
}

\section{rheumatoid knee}

\author{
Sergiu Andrei lordache***, Bogdan Șerban*****, Mihnea loan Gabriel Popa**, Adrian Cursaru** \\ *Department of Orthopaedics and Traumatology, University Emergency Hospital Bucharest, Romania \\ **Department of Orthopaedics and Traumatology, "Carol Davila" University of Medicine and Pharmacy, \\ Bucharest, Romania \\ ***Department of Orthopaedics, University Emergency Hospital Bucharest, Romania
}

Correspondence to: Sergiu Andrei lordache, MD, PhD, Department of Orthopedics and Traumatology, University Emergency Hospital Bucharest, 169 Splaiul Independenței Street, Code 050098, Bucharest, Romania, Mobile Phone: +40766 315 091, E-mail: sergiu.iordache92@yahoo.com

\section{Abstract}

Rheumatoid arthritis (RA) represents a condition that can erode cartilage and damage joints, leading to inflammation and loss of movement, characterized by inflammatory synovitis. While the widespread use of potent disease-modifying medications has increased opportunities for RA patients, orthopedic surgery and complete joint arthroplasty remain an important option in end-stage joint treatment. The knee is one of the most frequently affected joints in chronic rheumatoid arthritis patients. The severity of RA ranges from a moderate illness to a serious, rapidly progressing, destructive version, gradually leading to incessant pain and joint deformity. Despite recent advances in biological agents and therapeutic modalities in the field of rheumatology, certain patients with RA, who ultimately undergo joint surgery, tend to experience progressive joint damage. Though, TKA can be performed in these patients, increased complications and poorer outcomes may result after total knee arthroplasty, because of the particularities given by RA. They are associated with extended operating time, specifically resulting in increased infection, blood loss and deep vein thrombosis. However, because RA patients present additional risk factors for complications, certain critical preoperative examination and surgical aspects need to be considered in order to maximize TKA outcomes in this subgroup of patients.

Keywords: total knee arthroplasty, rheumatoid arthritis, flexion contracture, TKA complications, rheumatoid cysts, osteopenia

\section{Introduction}

Rheumatoid arthritis (RA) is a systemic pathology with polyarticular destruction, which has many extra-articular manifestations in most cases. Some of these are anemia and specific comorbidities, like cardiovascular diseases, which require careful preoperative evaluation in collaboration with specialists from many medical branches so that the results are optimal and, at the same time, the risk of side effects is reduced. 
Joint pain and physical disability represent the most commonly diagnosed conditions of RA patients, but their prognosis is largely determined by cardiovascular events, including venous thromboembolism (VTE). Compared with the general population, the risk of VTE is more than double in patients with RA. The incident rate in RA patients is calculated to be approximately 4 cases per 1000 person-years [1].

The pathogenesis of thrombotic susceptibility in RA is linked to different pathways and causal factors (hyperhomocyteinemia, inflammation, antiphospholipid antibodies), vascular defects, hypercoagulation and venous stasis, the three components of the Virchow triad are triggered in RA patients. Uncontrolled RA, identified as the need to alter the disease-modifying antirheumatic drugs (DMARD), raises the occurrence of VTE in observational studies [2].

Rheumatoid arthritis is a condition that has the capability to erode cartilage and damage joints, leading to inflammation and loss of movement, characterized by inflammatory synovitis. While the widespread use of potent disease-modifying medications has increased opportunities for RA patients, orthopedic surgery and complete joint arthroplasty remain vital in end-stage joint treatment [3].

Over half of the RA patients have previously been expected to undergo orthopedic surgeries, most commonly arthroplasty, during the duration of their disease. While total knee arthroplasty (TKA) and total hip arthroplasty (THA) rates have increased dramatically in the general population, contemporary RA surgery rates have declined or remained partly constant due to the impact of disease-modifying therapy (disease - modification of antirheumatic drugs [DMARD]).

The knee is one of the most frequently affected joints in chronic rheumatoid arthritis patients. The severity of RA ranges from a moderate illness to a serious, rapidly progressing, destructive version, gradually leading to incessant pain and joint deformity. Despite recent advances in biological agents and therapeutic modalities in the field of rheumatology, certain patients with RA, who ultimately undergo joint surgery, tend to experience progressive joint damage [4].

When synovectomy has little advantage in the advanced disease, total knee arthroplasty (TKA) has proven to be an effective intervention in RA patients that decreases knee pain and enhances physical function.

However, because RA patients have additional risk factors for complications, certain critical preoperative examinations and surgical aspects need to be considered in order to maximize TKA outcomes in this subgroup of patients $[\mathbf{1}, \mathbf{2}]$.

\section{Preoperative and postoperative considerations}

In rheumatoid arthritis, ipsilateral hip involvement is more common than in osteoarthritis [1]. Until total knee arthroplasty (TKA), the hip should always be extensively tested and, with a few exceptions, the hip should be replaced prior to the knee $[\mathbf{2}, \mathbf{5}]$. There are at least six reasons for this: the first is that it is better to overcome any hip-referred knee pain first. At times, due to the pain relief obtained by replacing the joint, knee arthroplasty can also be postponed. The hip joint should be treated conservatively in situations where it is critical to overcome the cause of the knee pain. Both the patient and the surgeon are more comfortable with the decision to start with the hip first if the pain relief is important.

In young patients with RA, the second explanation is particularly significant, since the patient has reasonably simple and painless hip surgery (compared with the knee surgery). On the opposite, the discomfort and painful 
recovery that the patient endures without a substantial benefit if the knee is operated first.

The fact that a patient can exercise a hip above a painful arthritic knee is a third factor, while exercising a knee below a painful, rigid arthritic hip is difficult. A stationary bicycle, for example, is extremely helpful during knee arthroplasty rehabilitation, but it is not important for hip replacement rehabilitation. If the hip above is painful and rigid, the use of a bicycle is not possible [3].

The fourth reason is to resolve the tension of muscles that cross both the hip and knee joint, especially the hamstrings. If, for example, both hip and knee have flexion contracture and the knee is operated on first, resolving that contracture, a subsequent hip replacement that lengthens the hip can retighten the hamstring muscle.

The fifth reason is also related to preoperative knee contracture [2]. At the time of hip arthroplasty, a contracted knee can be manipulated and casted to improve passive extension before the knee replacement. If epidural anesthesia is used, it can be maintained for several days and serial casts can be applied.

Finally, the sixth is that the prevention of a well-balanced TKA from twisting and torquing while dislocating and exposing a rigid hip for replacement makes sense.

In patients with RA, deep vein thrombosis (DVT) and pulmonary emboli occur less often than in patients with osteoarthritis [4]. This may be due partially to the fact that most RA patients need chronic use of antiinflammatory drugs that have a mild anticoagulation effect. It may also be intrinsically linked to the process of their disease $[6,7]$. The explanation is unclear, but it makes their needs for anticoagulation distinct from those of osteoarthritis patients.

In order to anesthetize the patient with rheumatoid arthritis, special precautions are also needed due to the rheumatoid involvement of the cervical spine and temporomandibular joint. To foresee the patient's specific needs, preoperative evaluation by the anesthesiologist is essential. To test the patient for $\mathrm{C}_{1}-\mathrm{C}_{2}$ subluxation, lateral cervical spine films with full flexion and extension may be necessary. Regional anesthesia is chosen because of the many possible complications involved with the administration of general anesthesia [8].

The surgical team also needs to be prepared for the eventual need of general anesthesia and intubation. The anesthesiologist should be experienced in the use of nasotracheal intubation procedures and a pediatric fiber optic laryngoscope should be prepared [9].

In patients with $\mathrm{RA}$, the risk of perioperative and late hematogenic infection is greater than in those with osteoarthritis, and the appropriate incidence is possibly $0.5 \%$. This complication can be solved using cement spacers with antibiotic loading, in order to achieve a higher rate of success in treating periprosthetic joint infections [10].

Due to the immunosuppressed state of these patients arising from the disease or drugs, late infection can occur at an elevated pace and since they also have several remote sites of chronic infection that can metastasize to the site of arthroplasty $[\mathbf{1 1}, \mathbf{1 2}]$. The most common sites are in the foot and the lower leg. Another potential site is the olecranon bursa.

\section{Intraoperative considerations}

It is possible for active rheumatoid synovitis to recur after TKA. When the patella has not been resurfaced and the residual cartilage remains in the knee joint, the potential is increased.

Even with TKA, which requires patellar resurfacing, the knee may return to active rheumatoid synovitis. These patients are 
difficult to diagnose, often having a high effusion acute appearance, a high sedimentation rate, and an aspirate with a high cell count. In this case, the most probable risk is infection, but active rheumatoid arthritis must also be considered. The count of cells per high-power field with a predominance of either polymorphonuclear cells or lymphocytes can be as high as 20,000 or 30,000 white cells. The proportion of polymorphonuclear cells will not, as would be typical of infection, be in the high gos. To confirm the diagnosis of a rheumatoid disease, percutaneous histologic synovial biopsy may be appropriate. This typically happens in the case of a rheumatoid flare with multiple joint involvement. An intraarticular corticosteroid injection will silence the process if infection has been ruled out. Open synovectomy will sometimes be necessary.

This raises the question of whether a synovectomy should be performed on the rheumatoid knee at the time of primary arthroplasty. At the time of replacement, it is recommended that this be performed uniformly in patients with active synovitis. Synovectomy is possibly not required if the disease is silent and the synovium is not inflamed $[13,14]$.

Rheumatoid arthritis is more likely to cause flexion contracture than osteoarthritis. The contracture is more likely to be caused by softtissue inflammation, whereas osteoarthritis flexion is typically caused by bony blocks. The following treatment recommendations for patients with rheumatoid arthritis were established after observing the results of many patients with serious contracture.

The distal femoral resection should be increased by $2 \mathrm{~mm}$ if the flexion contracture is between 15 and 45 degrees. This should be done to a complete maximum of $13 \mathrm{~mm}$ to prevent the femoral source of the collateral ligaments from being affected [15-17].
Preoperative manipulation and casting between 45 and 60 degrees of flexion contracture should be considered and a posterior cruciate ligament ( $P C L$ )-substituting technique should be always used. Preoperative manipulation and casting and a restricted prosthesis for flexion contracture greater than 60 degrees should be considered in order to overcome flexion gap laxity that may result from substantial elevation of the femoral joint axis [18].

For patients with inflammatory arthritis, the "rule of one third" should be adopted. According to it, under anesthesia, intraoperative correction only needs to be within one third of the preoperative contracture. With physical therapy, inflammatory disorder resolution, and sometimes the aid of manipulation and casting, the remaining one-third typically resolves.

Several additional points should be illustrated concerning the flexion contracture. If the patient has extreme bilateral flexion contracture, both knees should be corrected simultaneously to reduce the possibility of correction regression [19].

When the capsule is closed after an extreme flexion contracture has been reversed, the medial capsule should be relocated distally to the lateral capsule in order to prevent the initial lag of the extensor mechanism [20].

Zero degrees should be the amount of posterior slope added when dealing with an extreme flexion contracture. Each degree of slope built into the tibia generates or prevents the correction of a degree of flexion contracture.

Finally, following surgery, preventive steps should be taken, such as enabling the patient to have a roll under the ankle, but never under the knee. At night, a knee immobilizer will keep the patient from sleeping with flexed knees and reconstructing a contracture. A 
dynamic extension splint may be useful in refractory cases for both correcting and preserving extension [21].

To have adequate function, patients with rheumatoid arthritis need more knee flexion than those with osteoarthritis. To walk on level land, all individuals need 60 to 70 degrees of flexion. For ascending most stairs, ninety degrees of flexion is required. To descend stairs in a normal fashion, one hundred degrees of flexion is needed. To rise from a standard chair without pressing up using the upper extremities, one hundred and five degrees or more is required [22].

In patients with $R A$, the regular involvement of knees, elbows, wrists, and hands affects their ability to climb stairs or rise from a sitting position if they have insufficient knee flexion. The presence of the disease in the upper extremity can also enable them to use a platform crutch or walker that cradles the forearm with vertical grip handles, a platform crutch may typically be adjusted.

While small juxtaarticular cysts in osteoarthritis are not rare, they are more common in RA, and on the femoral or tibial side of the joint, very large cysts are occasionally present [23]. All cysts should still be free of soft tissue and grafted to seal the cyst wall from the cement interface with cancellous bone. In patients with RA, failure to do this leads to the risk of gradual demarcation at the interface of the cementcyst, leading to loosening. At the loosening interface, analysis of the tissue reveals histological characteristics consistent with those of chronic rheumatoid synovium.

With an impact grafting procedure, larger central defects may be treated [24]. In this process, the morselized bone graft is tightly packed around a long trial stem. The graft should be so tightly packed as to have structural integrity when the trial is removed. The stem of the component may either be press-fit or cemented, depending on the distal bone condition in the tibia. If the cemented technique is selected, prior to applying the bone graft, the canal is blocked with a cement restrictor.

It remains controversial whether to resurface the patella during TKA. In patients with RA, there is a disparity in long-term outcomes relative to osteoarthritis because the patella has not been resurfaced. Patients with RA have secondary patellar degeneration without resurfacing, and even have the risk of recurrent knee rheumatoid synovitis. Although some patients with non-patellar resurfacing RA have survived their third decade after TKA, the rule that patella should be resurfaced in all patients with rheumatoid arthritis, regardless of surgical findings, is adhered to $[\mathbf{2 5}, \mathbf{2 6}]$.

Rheumatoid disease-associated osteopenia can present difficulties during TKA $[27,28]$. Postoperative fractures of femoral stress after anterior notching are common in patients with RA. In these patients, the risk of stress fracture is so high that a long-stem femoral component should be considered if the cortex is substantially notched. Notching is more likely to happen when the smaller size is chosen for a femur that is between sizes.

Supracondylar fractures can occur when preparing the leg for surgery, when osteopenia is serious [29]. The hip above is rigid in these situations, and with too much force, the assistant lifting the leg could produce a supracondylar femoral fracture. Similarly, in patients with rheumatoid arthritis, supracondylar fractures occur during leg preparation for complete hip arthroplasty, when too much abduction force has been applied to the ankylosed hip.

During postoperative manipulation, fractures may also be created due to poor motion range after TKA.

Avulsion of the medial collateral ligament from its femoral origin due to medial retraction is another possible intraoperative complication in patients with extreme 
osteopenia and rheumatoid arthritis. The medial cortex that divides from the soft underlying cancellous bone is usually included in the avulsion. It is typically not a clinical concern, as troubling as this might be, since the soft tissue sleeve stays intact.

A complication of osteopenia is also an intraoperative fracture of the patella. After patellar preparation, this could occur, including the positioning of the holes for the fixation plugs. These holes are now stress risers, and a fracture can be propagated in a natural fashion simply by retracting against the patella. The fixation provided by the cemented patellar portion across the fracture can suffice if the fragments are minimally separated. The surgeon must consider cerclage wiring if the fragments are displaced [30].

\section{Conclusions}

To relieve pain and improve the physical function of patients with RA, TKA has been identified as one of the most effective treatment methods. Understanding the systemic nature of RA is important, since patients also have medical, anesthetic, and various musculoskeletal issues compared to OA patients.

Due to the slow healing nature of soft tissues, a higher rate of deep wound infection, extreme preoperative joint deformity and laxity, poor bone stock and other effects, complications after TKA may be more frequent and more severe in RA patients.

Subsequent tibia subluxation, proximal tibial bone deficiency combined with valgus deformity and external tibia rotation typically occur in RA knees with extreme flexion contracture, which may be partly due to contracture and traction of the biceps muscle and iliotibial tract.

The role of periarticular soft tissues in rheumatoid arthritis forms part of the constellation of pathology. Therefore, it is necessary to correct the deformity, to equalize the tension of the medial and lateral soft tissues and to accurately implant the components.

For the success of the operation, proper balancing of soft tissues in the form of ligament and capsular release at the time of arthroplasty is necessary. The correct orientation of the individual components and the resulting general alignment of the lower extremity in the RA knee with TKA in one step was especially important with respect to the remainder of the flexion in action.

The 2017 American College of Rheumatology and American Association of Hip and Knee Surgeons (ACR/ AAHKS) guideline for the perioperative management of antirheumatic drug therapy for adults undergoing elective THA and TKA was planned for use by clinicians and patients during the perioperative period. The included guidelines address the use of antiinflammatory drug therapy (including DMARDs, tofacitinib, biologic agents and glucocorticoids) in adults with RA, spondyloarthropathy (SpA), including ankylosing spondylitis (AS) and psoriatic arthritis (PsA), juvenile idiopathic arthritis (JIA) or systemic lupus erythematous (SLE), acknowledging that anti-inflammatory drugs are commonly used at the time of THA or TKA, and that infection rates and adverse events, including readmission, are higher in this population group.

Increased complications and poorer outcomes after TKA are associated with extended operating time, resulting specifically in increased infection, blood loss and deep vein thrombosis.

Though TKA can be performed in these difficult patients, complete intraoperative correction of extreme deformity of flexion has been difficult for orthopedic surgeons. Various strategies for resolving these deformities have 
been identified, including additional bone resection, release of ligaments, and the use of increased constriction prosthesis.

However, during surgery, an optimal soft tissue equilibrium is difficult to achieve.

In order to ensure the success of TKA procedures, proper soft tissue balance in the form of ligament and capsular discharge at the time of arthroplasty is necessary, which not only achieves a direct correction of bending contracture, but also effectively enhances the range of motion and functional recovery of the knee joint.

\section{Conflict of Interest statement}

The authors state no conflict of interest.

\section{Acknowledgements}

Professional editing, linguistic and technical assistance performed by Irina Radu, Individual Service Provider, certified translator in Medicine and Pharmacy (credentials: Eoo48/2014).

\section{Sources of Funding}

Not applicable.

\section{Disclosures}

None.

\section{References}

1. Goodman SM, Springer $B$, Guyatt $G$, Abdel MP, Dasa V, George M, Gewurz-Singer O, Giles JT, Johnson B, Lee $S$ et al. 2017 American College of Rheumatology/American Association of Hip and Knee Surgeons Guideline for the Perioperative Management of Antirheumatic Medication in Patients With Rheumatic Diseases Undergoing Elective Total Hip or Total Knee Arthroplasty. J Arthroplasty. 2017; 32(9):2628-38.

2. Lee $\mathrm{JK}$, Choi $\mathrm{CH}$. Total knee arthroplasty in rheumatoid arthritis. Knee Surg Relat Res. 2012; 24(1):1-6.

3. Conaty JP, Nickel VL. Functional incapacitation in rheumatoid arthritis: a rehabilitation challenge. A correlative study of function before and after hospital treatment. J Bone Joint Surg Am. 1971; 53:624-37.

4. Buchanan RR, Kraag G. Is there a lower incidence of deep venous thrombosis after joint replacement in rheumatoid arthritis? J Rheumatol. 1980; 7(4):551-4.
5. Goodman SM. Rheumatoid arthritis: preoperative evaluation for total hip and total knee replacement surgery. J Clin Rheumatol. 2013; 19(4):187-92.

6. Ketfi C, Boutigny A, Mohamedi N, Bouajil S, Magnan B, Amah G, Dillinger JG. Risk of venous thromboembolism in rheumatoid arthritis. Joint Bone Spine. 2020; 88(3):105122.

7. Mameli A, Barcellona D, Marongiu F. Rheumatoid arthritis and thrombosis. Clin Exp Rheumatol. 2009; 27(5):846-55.

8. Memtsoudis SG, Cozowicz C, Bekeris J, Bekere D, Liu J, Soffin EM, Mariano ER, Johnson RL, Hargett MJ, Lee $\mathrm{BH}$ et al. Anaesthetic care of patients undergoing primary hip and knee arthroplasty: consensus recommendations from the International Consensus on Anaesthesia-Related Outcomes after Surgery group (ICAROS) based on a systematic review and metaanalysis. Br J Anaesth. 2019; 123(3):269-87.

9. Wong PB, McVicar J, Nelligan K, Bleackley JC, McCartney CJ. Factors influencing the choice of anesthetic technique for primary hip and knee arthroplasty. Pain Manag. 2016; 6(3):297-311.

10. Cursaru A, Cretu B, Serban B, Lupu AG, lacobescu G, Popa M, Cursaru R, Cirstoiu C. Mechanical safety study and antibiotic-loaded polymethylmethacrylate spacers threshold, manufactured intraoperatively, in orthopaedic surgery. Mat Plast. 2020; 4:317-324.

11. Ravi B, Croxford R, Hollands S, Paterson JM, Bogoch E, Kreder $\mathrm{H}$, Hawker GA. Increased risk of complications following total joint arthroplasty in patients with rheumatoid arthritis. Arthritis Rheumatol. 2014i 66(2):254-63.

12. Cordtz R, Odgaard A, Kristensen LE, Overgaard S, Dreyer L. Risk of medical complications following total hip or knee arthroplasty in patients with rheumatoid arthritis: A register-based cohort study from Denmark. Semin Arthritis Rheum. 2020; 50(1):30-35.

13. Liu P, Lu F, Chen J, Xia Z, Yu H, Zhang Q, Wang W, Guo $W$. Should synovectomy be performed in primary total knee arthroplasty for osteoarthritis? A meta-analysis of randomized controlled trials. J Orthop Surg Res. 2019; 14(1):283.

14. Zhao ZQ, Xu J, Wang RL, Xu LN. The efficacy of synovectomy for total knee arthroplasty: a metaanalysis. J Orthop Surg Res. 2018; 13(1):51.

15. Matsui $Y$, Matsuura $M$, Minoda $Y$, Nakagawa $S$, Okajima $Y$, Kobayashi A, Inori F. Intraoperative manipulation for flexion contracture during total knee arthroplasty. J Orthop Surg (Hong Kong). 2019; 27(1):2309499019825574.

16. Okamoto S, Okazaki K, Mitsuyasu H, Matsuda S, MizuUchi H, Hamai S, Tashiro Y, Iwamoto Y. Extension gap needs more than 1-mm laxity after implantation to avoid post-operative flexion contracture in total knee arthroplasty. Knee Surg Sports Traumatol Arthrosc. 2014i 22(12):3174-80.

17. Kim SH, Lim JW, Jung HJ, Lee HJ. Influence of soft tissue balancing and distal femoral resection on flexion contracture in navigated total knee arthroplasty. Knee Surg Sports Traumatol Arthrosc. 2017; 25(11):3501-07. 
18. Song SJ, Park CH, Bae DK. What to know for selecting cruciate-retaining or posterior-stabilized total knee arthroplasty. Clin Orthop Surg. 2019; 11(2):142-150.

19. Peters CL. Soft-tissue balancing in primary total knee arthroplasty. Instr Course Lect. 2006; 55:413-7.

20. Hwang YS, Moon KP, Kim KT, Kim JW, Park WS. Total knee arthroplasty for severe flexion contracture in rheumatoid arthritis knees. Knee Surg Relat Res. 2016; 28(4):325-9.

21. An VVG, Scholes CJ, Fritsch BA. Factors affecting the incidence and management of fixed flexion deformity in total knee arthroplasty: A systematic review. Knee. 2018; 25(3):352-9.

22. Yan D, Yang J, Pei F. Total knee arthroplasty treatment of rheumatoid arthritis with severe versus moderate flexion contracture. J Orthop Surg Res. 2013; 8:41.

23. Scott DL, Wolfe F, Huizinga TW. Rheumatoid arthritis. Lancet. 2010; 376(9746):1094-108.

24. Brick GW, Scott RD. The patellofemoral component of total knee arthroplasty. Clin Orthop Relat Res. 1988; (231):163-78.

25. Forster MC. Patellar resurfacing in total knee arthroplasty for osteoarthritis: a systematic review. Knee. 2004; 11(6):427-30.

26. Fu Y, Wang G, Fu $Q$. Patellar resurfacing in total knee arthroplasty for osteoarthritis: a meta-analysis. Knee Surg Sports Traumatol Arthrosc. 2011; 19(9):1460-6.

27. Adami G, Fassio A, Rossini M, Caimmi C, Giollo A, Orsolini G, Viapiana O, Gatti D. Osteoporosis in Rheumatic Diseases. Int J Mol Sci. 2019; 20(23):5867.

28. Ostrowska $M$, Maśliński W, Prochorec-Sobieszek $M$, Nieciecki M, Sudoł-Szopińska I. Cartilage and bone damage in rheumatoid arthritis. Reumatologia. 2018; 56(2):111-120.

29. Hoffmann MF, Jones CB, Sietsema DL, Koenig SJ, Tornetta 3 rd $\mathrm{P}$. Outcome of periprosthetic distal femoral fractures following knee arthroplasty. Injury. 2012; 43(7):1084-9.

30. Vakharia AM, Cohen-Levy WB, Vakharia RM, Sodhi N, Mont MA, Roche MW. Perioperative complications in patients with rheumatoid arthritis following primary total knee arthroplasty: An analysis of 102,898 patients. J Knee Surg. 2019; 32(11):1075-80. 